\title{
Research challenges in QoS routing
}

\author{
X. Masip-Bruin ${ }^{\mathrm{a}, *}$, M. Yannuzzi ${ }^{\mathrm{b}}$, J. Domingo-Pascual $^{\mathrm{a}}$, A. Fonte $^{\mathrm{b}}$, M. Curado $^{\mathrm{b}}$, \\ E. Monteiro ${ }^{\mathrm{b}}$, F. Kuipers ${ }^{\mathrm{c}}$, P. Van Mieghem ${ }^{\mathrm{c}}$, S. Avallone ${ }^{\mathrm{d}}$, G. Ventre ${ }^{\mathrm{d}}$, \\ P. Aranda-Gutiérrez ${ }^{\mathrm{e}}$, M. Hollick ${ }^{\mathrm{f}}$, R. Steinmetz ${ }^{\mathrm{f}}$, L. Iannone ${ }^{\mathrm{g}}$, K. Salamatian ${ }^{\mathrm{g}}$ \\ ${ }^{a}$ Computer Architecture Department, Technical University of Catalunya (UPC) Agda. Victor Balaguer, s/n, O8800 Vilanova i la Geltrú, \\ Barcelona, Catalunya, Spain \\ ${ }^{\mathrm{b}}$ Laboratory of Communications and Telematics, DEI/CISUC, University of Coimbra, Polo II, $3030-290$ Coimbra, Portugal \\ ${ }^{\mathrm{c}}$ Faculty of Electrical Engineering, Mathematics and Computer Science, Delft University of Technology, P.O. Box 5031, 2600 GA Delft, The Netherlands \\ ${ }^{\mathrm{d}}$ Dipartimento di Informatica e Sistemistica, Università degli Studi di Napoli 'Federico II', Via Claudio 21, 80125 Napoli, Italy \\ 'Telefónica I+D, Emilio Vargas, 6, 28043 Madrid, Spain \\ ${ }^{\mathrm{f}}$ Multimedia Communications Lab (KOM), Department of Electrical Engineering and Information Technology, Technische Universität Darmstadt, \\ Merckstr. 25, 64283 Darmstadt, Germany \\ ${ }^{\mathrm{g}}$ LIP6/CNRS, Université Pierre et Marie Curie, 8, rue du Capitaine Scott, 75015-Paris, France
}

Received 3 June 2005; accepted 3 June 2005

Available online 11 July 2005

\begin{abstract}
Quality of Service Routing is at present an active and remarkable research area, since most emerging network services require specialized Quality of Service (QoS) functionalities that cannot be provided by the current QoS-unaware routing protocols. The provisioning of QoS based network services is in general terms an extremely complex problem, and a significant part of this complexity lies in the routing layer. Indeed, the problem of QoS Routing with multiple additive constraints is known to be NP-hard. Thus, a successful and wide deployment of the most novel network services demands that we thoroughly understand the essence of QoS Routing dynamics, and also that the proposed solutions to this complex problem should be indeed feasible and affordable. This article surveys the most important open issues in terms of QoS Routing, and also briefly presents some of the most compelling proposals and ongoing research efforts done both inside and outside the E-Next Community to address some of those issues.
\end{abstract}

(C) 2005 Elsevier B.V. All rights reserved.

Keywords: QoS Routing; intra/inter-domain routing; multicast routing; routing optimization

\section{Introduction}

The concept of Quality of Service (QoS) in communication systems is closely related to the network performance of the underlying routing system. To establish a common understanding for network QoS and particularly QoS

\footnotetext{
* Corresponding author. Tel.: +34 93896 7767; fax: +34 938967700 .

E-mail addresses: xmasip@ac.upc.edu (X. Masip-Bruin), yannuzzi@ ac.upc.edu (M. Yannuzzi), jordid@ac.upc.edu (J. Domingo-Pascual), afonte@dei.uc.pt (A. Fonte), marilia@dei.uc.pt (M. Curado), edmundo@ dei.uc.pt (E. Monteiro), F.A.Kuipers@ewi.tudelft.nl (F. Kuipers), P. VanMieghem@ewi.tudelft.nl (P. Van Mieghem), stavallo@unina.it (S. Avallone), giorgio@unina.it (G. Ventre).
}

0140-3664/\$ - see front matter (C) 2005 Elsevier B.V. All rights reserved. doi:10.1016/j.comcom.2005.06.008
Routing we depart from the ITU's definition of Quality of Service [1].

Definition: 'Quality of Service-the collective effect of service performance which determines the degree of satisfaction of a user of the service.'

Fig. 1 shows the four major building blocks introduced in [1]: quality of service, serveability, trafficability performance, and dependability. To allow for implementation, the high-level concept of QoS can be mapped to service related primitives as described with the concept of serveability. The service performance is directly affected by the network performance. This ability of the network to meet the traffic demands is described by the concept of trafficability performance. Finally, dependability is a critical point impacting on the whole QoS network performance.

Routing can decisively contribute to the provision of QoS, and to the improvement of traffic performance and 


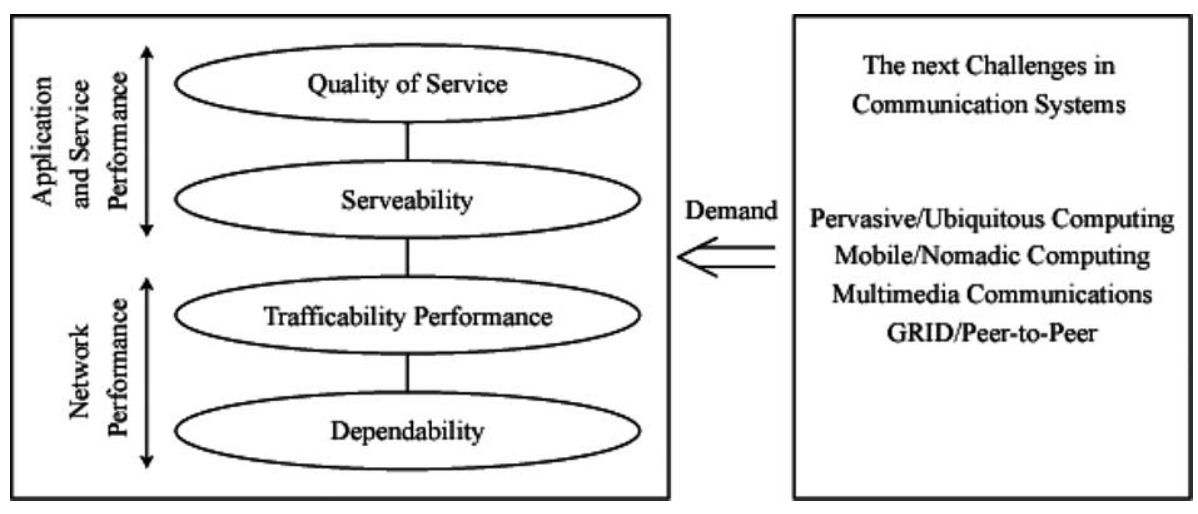

Fig. 1. Main QoS building blocks according to ITU [1].

dependability in the ITU model. Although the merit of QoS Routing has long been recognized [2], a full-scale deployment is still missing. In this article we present an accurate description of the current state-of-the-art and enumerate the main open QoS Routing issues where significant effort and research is needed.

Next, we briefly introduce the reader to the main open QoS issues focussing on QoS Routing, writing down the main algorithmic, dynamic, architectural and dependability aspects. Subsequently these open issues are discussed in detail.

\subsection{Motivation for QoS routing}

It is fair to state that the concept of Quality of Service (QoS) with its multidimensional service requirements was born in the late 1980 with the advent of ATM. Some years ago, QoS has been introduced in the Internet by a series of IETF contributions like Intserv, Diffserv, RSVP and MPLS. Currently, the IETF working group on traffic engineering is continuing to shape QoS induced features from the network provider's perspective. The interactivity of multimedia communication in the Internet is still increasing: real-time communication and QoS-awareness are regarded as valuable. Today, it is unclear what the role of QoS will be in newer types of networking such as mobile ad-hoc networks, sensor networks, WIFI and UMTS, grid computing, and overlay networking. In wired networks and especially in traditional telephony, network operators are facing the problem of replacing their relatively old classical telephony equipment, since the end of lifetime of switching fabrics is looming at the near horizon (2010). Their concern is the question whether it is possible or not to offer large-scale telephony (VoIP) over the current Internet with the conservation of the accustomed toll quality. In spite of the apparent importance of QoS, there does not seem to exist yet a business model for a QoS aware Internet. Perhaps the main importance of QoS lies in its lever function between economy (pricing) and technology (QoS Routing, QoS control, and QoS network management). But, undoubtedly the main disadvantage of QoS is the notorious complexity, which causes that QoS will only be implemented abundantly if we fully understand the QoS dynamics and can demonstrate its feasibility (in practice) and the associated economic gain.

The IETF QoS Routing working group was established as a continuation of the Birds of a Feather (BOF) session held at the IETF in June 1996 to discuss issues in Quality of Service Routing. The IETF QoS Routing working group has been stopped in the late $1990 \mathrm{~s}$, mainly because the thorough understanding of the problem was still lacking. The moral seems to be that a theory and conceptual understanding of the problem is needed before the standards and not vice versa. Nevertheless, QoS Routing is a logically required architectural functionality, because all current IETF standards rely on traditional QoS-unaware routing. From this perspective, QoS Routing is the missing piece in a full-fledged QoS architecture for the Internet.

A conceptual difficulty with QoS in general starts already with the definition, and the same holds for a subpart of QoS, QoS Routing, to which this article is devoted. If we take the viewpoint that routing consists of a routing algorithm (static) and routing protocol (dynamics), then a QoS Routing algorithm solves the Multi-Constrained (Optimal) Path $(\mathrm{MC}(\mathrm{O}) \mathrm{P})$ routing problem. In the MCP problem, each link $u_{-} v$ in a given graph is characterized by a link weight vector $w(u \rightarrow v)=\left[w_{1}, w_{2}, \ldots, w_{m}\right]$ with $m$ positive real numbers $w_{i}\left(u_{-} v\right) \geq 0$ as components. The MCP problem asks for a path $P$ from a source node to a destination node that satisfies Eq. (1) for all $1 \leq i \leq m$ QoS metrics, where $L_{i}$ are the QoS constraints on the path.

$w_{i}(P) \stackrel{\text { def }}{=} \sum_{(u \rightarrow v) \in P} w_{i}(u \rightarrow v) \leq L_{i}$

A path that satisfies all $m$ constraints is often referred to as a feasible path. There may be many different paths in the graph that satisfy the constraints and, therefore, it might be desirable to retrieve the path with smallest length $l(P)$ from the set of feasible paths. The problem that additionally optimizes some length function $l(P)$ is called the MultiConstrained Optimal Path (MCOP) problem. In addition to satisfying Eq. (1), the MCOP problem minimizes some 
length criterion such that $l(P) \leq l\left(P^{\prime}\right)$, all paths $P^{\prime}$ between source and destination. A flexibility in the $\mathrm{MC}(\mathrm{O}) \mathrm{P}$ problem is the length criterion $l(P)$ - the cost optimization functionwhich only needs to obey the properties of a norm of a vector. A difficulty of the $\mathrm{MC}(\mathrm{O}) \mathrm{P}$ problem is that it is NPcomplete [3]. This classification essentially means that the time required to solve the $\mathrm{MC}(\mathrm{O}) \mathrm{P}$ problem exactly cannot, in the worst case, be upper-bounded by a polynomial function. Therefore the $\mathrm{MC}(\mathrm{O}) \mathrm{P}$ problem has been interpreted as intractable, which, in turn, has spurred the proposals of many heuristics. Only a few exact QoS Routing algorithms such as SAMCRA (Self-Adaptive Multiple Constraints Routing Algorithm [5]) exist. Although QoS Routing algorithmic issues still require attention, the larger part seems to be reasonably well understood.

The second component in QoS Routing, the QoS Routing protocol responsible for information exchange and for routing dynamics, is believed to be a far more difficult problem as outlined below. In short, the QoS Routing protocol consists of all the actions that inform individual nodes with a consistent and updated view on the network and the link weight structure.

Being the missing piece in the IETF QoS architectures and needing solutions for the $\mathrm{MC}(\mathrm{O}) \mathrm{P}$ problem and for the routing information dissemination protocol QoS Routing is definitely an excellent research subject in the area of computer networks. In order to substantiate this statement, the following subsections present some topics that deserve further study.

\subsection{Algorithmic aspects in QoS routing}

As stated in the previous Section, the algorithmic problem in QoS Routing, called the $\mathrm{MC}(\mathrm{O}) \mathrm{P}$ problem is NP-complete. Some of the proposed heuristics only target special cases of the $\mathrm{MC}(\mathrm{O}) \mathrm{P}$ problem. For instance, when bandwidth is one of the constraints that must be satisfied by the path computation algorithm, the MCP problem is defined as a Bandwidth Restricted Path (BRP) problem [6-10]. Another popular subproblem is called Restricted Shortest Path (RSP) problem [11-13]. In this case, all the paths that satisfy the constraint associated with one of the two metrics are computed and then the shortest path according to the second metric is selected. A straightforward method for heuristically solving the general MCP problem is via Metrics Combination (MC) [4,14-16]. By combining a set of QoS metrics in a single metric, it is possible to use existing polynomial-time path computation algorithms, such as Bellman-Ford or Dijkstra.

Of course, when using exact QoS algorithms, QoS guarantees can be made, which is not possible (or can only be approximated) with heuristics. It is therefore desirable to be exact, but this may come at a high price in terms of execution time. Fortunately, the theory of NP-completeness is based on a worst-case analysis, and knowing what kind of network scenarios constitute a worst case is valuable (both in theory and in practice). Kuipers and Van Mieghem have distinguished in [17] several conditions that must hold simultaneously in order for worst cases to emerge: (1) the underlying topology must have a large expected hop-count, (2) the link weights can grow arbitrarily large or have an infinite granularity, which is not the case in practice, (3) there is a very negative correlation among the link weights, and (4) the constraint values are not too large nor too strict. These conditions are highly unlikely to reflect typical (practical) cases, suggesting that exact QoS Routing is feasible in practice.

In [18] and [19] the most relevant of QoS algorithms are described and evaluated via simulations: SAMCRA performed best. However, SAMCRA may possibly be improved, which requires a good understanding of the complexity of QoS Routing itself. If it can be demonstrated (rigorously) that QoS Routing possesses an acceptable complexity (hence, feasible in practice), then it may be regarded as a fundamental cornerstone and the consequences may be far-reaching.

To conclude, concerning the algorithmic aspects of QoS Routing several questions are still open. Some of them are the following:

- Can the computational efficiency of exact QoS Routing algorithms such as SAMCRA still be improved? If so, how can it be done?

- Can topologies be pruned a priori in order to reduce the computational effort?

- Can new computationally more efficient data structures be used instead of the Fibonacci-heaps used in SAMCRA [20]?

- Can 'NP-complete' topologies be detected a priori [17] and [21]? If so, by assigning proper link weights, network management may avoid these hard cases.

- Extensions to multicast QoS Routing such as MAMCRA [22] need to be explored further.

- Extensions to link-disjoint QoS Routing such as DIMCRA [18] need to be explored further.

The answer to the above questions is the subject of ongoing research work.

\subsection{Dynamic aspects in QoS routing}

The current toughest problem that hampers the implementation of QoS in the Internet concerns the QoS Routing protocol. To enable QoS Routing, it is necessary to implement state-dependent, QoS-aware networking protocols. An example of such a protocol is PNNI, which uses link-state routing, in which every node tries to acquire a 'map' of the underlying network topology and the available resources via flooding. The available resources on a link are expressed by values, called link weights. Although simple and reliable, flooding involves unnecessary 
communications and causes inefficient use of resources, particularly in the context of QoS Routing that requires frequent distribution of multiple, dynamic parameters. Monitoring any change along the Internet is simply not possible and even not desirable, because not all changes are important. Two possible changes are considered:

(1) Infrequent changes due to joining/leaving of nodes. In the current Internet, only this kind of topology changes is considered. Its dynamics are relatively well understood.

(2) Frequent changes, which are typically related to the consumption of resources or to the traffic flowing through the network.

The link weight coupling to state information seriously complicates the dynamics of flooding because, contrary to infrequent changes, the flooding convergence time can be longer than the change rate of some metric (such as available bandwidth). The identification of the QoS characteristics and their characterization is determinant to the conception of QoS-aware routing protocols. QoS characteristics used to support the routing decision usually include bandwidth, loss rate, delay and jitter. Choosing the metrics upon which to base the routing decision is one of the main issues that must be addressed in a routing strategy because it determines simultaneously the characteristics that are offered to traffic and the complexity of the path computation algorithm. The selection of metrics must be done in order to increase the network self-awareness and service awareness.

The definition of issues related to metrics should contribute to increase the self-awareness and service awareness through the definition of the decisions concerning metrics selection and the mechanisms for metrics manipulation. The computation of QoS-aware paths requires that the routers obtain information about the state of the network in terms of the chosen metrics. The state of the network is composed of the local state of each node and of the global state that pertains to existing paths. The global state maintained by each node is obtained by the distribution of local states of the nodes that constitute the network.

An optimal update strategy for the infrequent changes is highly desirable in future multimedia networks that are characterized by the broad variability in traffic profiles and QoS requirements. No detailed update strategy for the infrequent changes has been published yet, although some descriptive papers have already appeared. Therefore the following points still deserve attention:

- What are the link weights $w_{1}, w_{2}, \ldots, w_{m}$ ? Type of metrics, number of metrics or relative significance of metrics.

- What is the influence of variations or inaccuracies (instabilities) on the link weights on the properties of the shortest (QoS) path? How can we handle it?
- Precision of metrics on the routing decision place

- What is the impact of aggregating routing information on the processing overhead? Would it be possible to reduce this processing overhead by means of path pre-computation?

- How do we determine, update and flood the link weight vectors? Is prediction possible?

- Proof of the QoS Routing conjecture 'QoS Routing is near to optimal load balancing'. More precisely, consider a network that is loaded by reserving resources per source-destination pair using an exact QoS Routing algorithm on an instantaneously updated topology. If a steady state is reached, we conjecture that the consumption of the network resources will be close to an optimally loaded network. If true, dynamic QoS Routing would imply load balancing and load balancing need not be treated as a separate optimization step.

Further there is a topology range of interest: not all details of the entire global Internet are needed to determine a path from A to B. A sub-network encompassing A and B seems sufficient. In this respect, the properties of a network topology are very important. The Internet is shown to possess a power-law like degree distribution, while Ad-Hoc networks may vary from lattice structures to random graphs. Since paths strongly depend on both link weight structure and graph properties, the network dynamics will depend on these factors, even to the extent that some control strategies successful in a certain class of graphs may not work properly in other graphs.

\subsection{Architectural aspects in QoS routing}

The combination of QoS Routing algorithm and QoS Routing protocol forms the basis for a QoS architecture for the Internet. However, several issues are still open:

- Hierarchical QoS Routing: intra- and interdomain QoS Routing

- How do we manage the QoS Routing fairness (coexistence of QoS flows/classes and best-effort)?

- How do we design a future save QoS Routing architecture (=both algorithm and protocol)?

- What is the level of detail required of the packetlevel? What of the flow level? (=Architectural Issues \& current RFCs)

- We need test bed verifications of proposed QoS Routing protocols and the influence of other control mechanisms as e.g. TCP.

- QoS Routing in wireless and Peer-to-peer networks.

- QoS Routing protocols are mostly evaluated by simulation, but how far can simulation go? Is a prototype implementation necessary? 


\subsection{Dependability in $Q o S$ routing}

The origins of dependability can be traced back to the early days of computing and communication as described in [23]. In the context of the early and pioneering work of Babbages, Larnder in 1834 proposed to eliminate errors in computation by using separate and independent computers and even more decisive by using different computation methods. Later, the first electronic computers and communication systems used highly unreliable components. As a result the research focussed on enhancing the reliability and dependability of operation-a first step towards QoS. Basic theories of redundancy to enhance the reliability of logical structures and to enhance the quality of communication have been developed from von Neumann, Moore, Shannon and their successors and are still the basis for our work. Today, the fundamental concept of dependability in computer/communication systems is discussed from a technical perspective in various research groups and committees including the joint initiative of the International Federation for Information Processing (IFIP WG 10.4 on dependable computing and fault tolerance) and the IEEE computer society (IEEE TC-FTC-technical committee on fault-tolerant computing) IEEE [24].

We now focus on the aspects of dependability that are closely related to communication networks and especially the Internet. Dependable operation of the routing system is part of the QoS Routing agenda since the early days of the Internet. For example, the predecessor of the Internet, the ARPANET, suffered from catastrophic failures because of its routing protocol, which could only be repaired with manual intervention (see, for example [25] for details of this malfunction). Based on this experience, the Internet community decided to require routing protocols to fulfil some basic dependability criteria such as, for example, the ability of the protocol to stabilize after the failure condition is removed (self-stabilization). Influenced by the failure of the ARPANET, routing protocols for the Internet have been kept very simple, though. Even today and despite the fact of high application QoS demands, the Internet lives without QoS and without QoS-capable routing mechanisms. We conclude that the dependability and survivability of the core transport functionality even under extreme conditions makes up one important point in the QoS Routing open agenda. We define routing dependability to be:

'Routing dependability is the trustworthiness of a routing system such that reliance can justifiably be placed on the consistency of behaviour and performance of the routing service it delivers.' [26]

To be able to design dependable QoS Routing systems, it is necessary to better understand the dimensions of routing dependability. These dimensions are not fixed, however, but are influenced by the characteristics of the investigated network. For the example of mobile and wireless communications (see also Section 5 of this article) we find some important characteristics to influence routing dependability to be [26]:

- User and end system mobility.

- The wireless nature of the communication channel.

- The routing strategies/algorithms and routing protocols, i.e., the adaptation to changing network conditions on various time-scales as well as the overhead induced.

- The infrastructure-based, infrastructure-less, or hybrid nature of the routing systems.

- The limitations in energy-resources.

- Asymmetric capabilities of nodes in heterogeneous networks.

- Cooperation vs. non-cooperation of network nodes in ad hoc networks.

- External forces, like environmental conditions.

\subsection{Outline}

We have described the main open QoS issues and have clearly justified the need for QoS Routing, given that the main goals of this article are in fact both to state those open issues as well as to present the most recent and significant contributions (some of them from E-Next partners) addressing such issues. This article is split in different sections covering a significant spectrum of the recent and future work to be done in QoS Routing. Section 2 focuses on intra-domain routing, describing recent work and new proposals in such a routing scenario. Section 3 extends the QoS Routing problem to inter-domain routing, also describing the most recent activities carried out on this topic. Optimization issues are analyzed in Section 4. Afterwards, in Section 5 we extend QoS Routing to wireless networks. Being aware of the main target of this article, we introduce in Section 6, as a brief summary, the main points of interests of the partners of E-Next involved in the writing of this article. Finally, Section 7 concludes the article.

\section{Intra-domain issues}

Internet routing can be uncoupled into two distinct planes, each of which has very different characteristics and goals, namely intra-domain routing and inter-domainrouting. On the one hand, intra-domain routing handles routing within a single network or administrative domain. Each administrative domain is free to choose the intradomain routing protocol to be utilized within its network, according to its own preferences and needs.

Two types of intra-domain routing protocols are available at present, that is link-state routing protocols and distance-vector routing protocols. Link-state protocols distribute the entire network topology to all routers within 
the domain, and the decision process to select the best path to reach any given destination inside this domain is based on Dijkstra's shortest path algorithm. Alternatively, in distance-vector routing protocols the routers lack of the entire network topology and the selection of the best path is based on the Bellman-Ford routing algorithm. At present, the most widely deployed intra-domain routing protocol is a link-state protocol, i.e. the Open Shortest Path First (OSPF) [27].

On the other hand, across the administrative domain boundaries an inter-domain routing protocol is used in order to exchange reachability information, and to select the best path to reach any given destination according to each domain's specific policies and needs. In contrast to the intradomain case, for inter-domain routing there is a de-facto standard routing protocol, i.e. the Border Gateway Protocol (BGP) [28]. BGP is a path-vector routing protocol, which for scalability reasons is only aware about the interconnections between the different administrative domains. In other words, BGP does not manage or exchange any kind of intradomain information, so the internal state of the network in any administrative domain is not revealed by BGP. In summary, whereas intra-domain routing manages the selection of the best path within a single administrative domain, inter-domain routing is what holds the Internet as a single unit.

In this section we focus on intra-domain QoS Routing, while inter-domain QoS Routing will be addressed in the next section. Main issues when selecting a path, such as complexity (routing overhead), routing information inaccuracy and routing stability are analyzed below. Then, multicast routing, link-disjoint routing and a predictionbased routing approach are also covered at the end of this section.

\subsection{Path selection algorithms for QoS routing}

The approaches used by the QoS Routing algorithms that compute multi-constrained paths usually follow a trade-off between the optimality of the paths and the complexity of the algorithm. The research of new path computation algorithms that improve complexity and the quality of the paths is thus still an open issue in the field of QoS Routing.

\subsubsection{Algorithmic and dynamic QoS routing overhead}

The objectives of QoS Routing protocols may be compromised by the additional burden they impose in the network. The weight introduced by QoS Routing approaches includes the following:

- Processing overhead due to more complex and frequent computations

- The additional storage needed to support QoS Routing protocols.
- The communication overhead caused by the increase on the amount of routing information exchanged within the network.

It is worth noting that all these factors closely impact on the network scalability, i.e. scalability becomes an issue that must be addressed by any QoS Routing proposal.

The processing overhead caused by QoS Routing is mainly due to two factors, namely, path computation algorithm complexity and the frequency of path computation. As the number of constraints that need to be satisfied by the routing algorithm increases, the complexity of the path computation algorithm becomes higher, demanding more processing resources. Then, in the case of path precomputation approaches, the QoS paths installed on the routing table must be up-to-date according to the state of the network, and in the case of on-demand path computation, the paths must be computed upon the reception of connection requests. In any of these routing styles, the path computation algorithm must be applied more often than in traditional routing protocols, therefore requiring more processing resources. Although most QoS Routing proposals use on-demand path computation, this routing style suffers from two drawbacks. Firstly, it introduces some delay before the forwarding of traffic starts. Secondly, it requires the application of the path computation algorithm for each connection request, introducing additional processing overhead on the routers, especially when the arrival rate of connection requests is high. The pre-computation of paths is the alternative approach to handle the problem of the processing overhead associated with on-demand path computation at the expense of the eventual inaccuracy of the routing decision [7] and [29].

QoS Routing raises router storage requirements due to the increased amount of information about the state of the network that is kept in the router and due to the size of the QoS Routing tables. The information kept in routers includes the metrics that describe the state of the links in the network, such as delay, loss rate and utilization, and statistical data about the traffic mix forwarded through the router. The size of QoS Routing tables is determined by the type of traffic classification used, and can vary from only one entry for each destination in the network, as in traditional routing, up to one entry for each active flow. Even though QoS Routing needs more storage resources, the amount of storage needed is affordable by the memory capacity of actual routers and, thus, it is not a severe constraint for the deployment of QoS Routing solutions in networks [30].

The communication overhead of QoS Routing protocols can have several sources, depending on the type of approach followed. In the case of link-state protocols, the communication overhead is due to the load of the flooding process used in the exchange of link-state information among the routers in the network. Since the routers need to have an upto-date view of the network state in order to support correct 
routing decisions, the state information needs to be distributed frequently and thus can highly increase the communication overhead. The impact of this problem depends on factors such as the number of QoS metrics, the timescale of the routing decision, the routing model and the instant of path computation. Probe-based QoS Routing protocols can also introduce communication overhead in the network. In this case, the overhead is caused by the probe messages that are used to collect the state of the links in the network or to examine alternate paths needed for specific connection requests. The amount of communication overhead due to probe messages depends on the number of probe messages issued, on their size and on the frequency of their emission. The signalling messages issued by QoS Routing protocols that perform on-demand path computation and path setup are another factor that contributes to the communication overhead. The signalling messages are used to request the computation of specific paths for ondemand path computation and to install the corresponding QoS routes on nodes along the QoS paths. These approaches are responsible for increasing both communication and processing overhead, especially in the presence of a large amount of short lived flows [31].

The reduction of the communication overhead can be achieved in two complementary ways. The first concerns the limitation of the amount of routing messages, by limiting the frequency of the emission of updates and by limiting the number of routers that are eligible to receive updates. The distribution of updates could be made as soon as a state change occurs in the network. However, this approach would lead to an excessive communication weight on the network and could also originate instability. Instead of distributing updates immediately after a change, the instant of distribution is generally controlled by triggering policies [6]. The process of flooding used by link-state protocols causes a burden that can consume an excessive amount of resources in the network, especially when used in QoS Routing schemes. Selective flooding solutions aim at reducing the overhead caused by the emission of link-state updates throughout the network [32].

The second is the reduction of the size of routing messages. The advertisement of quantified metrics, instead of the advertisement of instantaneous values, is a common approach to avoid the excessive communication cost of dynamic routing protocols [33]. The hierarchical organization of networks allows for the aggregation of routing information between the different levels and reduces the amount of information that must be distributed and stored, while decreasing the number of routers involved in the exchange of routing information. The conception of strategies for routing information aggregation must have in consideration the degree of aggregation and the resulting routing performance [34-36].

As the size of the networks grows, the weight caused by QoS Routing solutions may become excessive, affecting traffic performance. Scaling issues are related to the amount of information that flows in the network, to the complexity of the path computation algorithm, and to the amount of information stored and processed in routers. The mechanisms that can be used to control the routing overhead and contribute to scalability include metrics quantification and triggering policies, selective flooding, information aggregation and path pre-computation.

The overhead associated with QoS Routing is an important limitation to its deployment. Namely, the flooding process used to distribute the state of the network is one important factor in QoS Routing overhead. Mechanisms used to overcome the cost of QoS Routing, such as the ones that limit the frequency of the emission of updates, introduce new problems, namely routing information inaccuracy. Thus, the conception of new QoS Routing schemes that do not rely on flooding or that overcome its flaws is an issue that needs further investigation.

\subsubsection{QoS routing under inaccurate information}

The use of inaccurate routing information by path computation algorithms can severely damage the performance of QoS Routing protocols. Therefore, it is desirable that the state kept at all routers remains up-to-date and that it reflects the complete and detailed state of the network. However, there are several factors that prevent the fulfilment of this goal, as described bellow.

The low frequency of the distribution of routing linkstate updates is one source of inaccuracy of routing information [30]. In situations where the distribution of routing information is done periodically, the changes in network state that occur between two update instants are not spread, and thus the paths in use remain the same, leading to incorrect traffic distribution in the network. A similar situation occurs when the control of the distribution of routing messages is made by update policies, such as threshold-based or class-based. If the parameters that control the update policy are configured with low sensitivity in order to limit the amount of communication overhead, the link-state information in the routers becomes stale and the path computation algorithm may make wrong routing decisions.

The proposals that handle state information inaccuracy must deal with a trade-off between the protocol overhead needed to keep the state information up-to-date and the inaccuracy that arises with the limitation of the emission of updates. Therefore, schemes to overcome inaccuracy caused by out-of-date link-state information have two main objectives, namely, to improve protocol performance when there is inaccuracy in routing information and to reduce the protocol overhead associated with frequent distribution of updates.

Information aggregation in hierarchical networks is also an important factor of routing information inaccuracy in large networks [37]. Even in intra-domain routing protocols, such as OSPF, the routers are grouped in areas to allow for scalability and faster convergence times [27]. In 
hierarchical networks the routing metrics of physical links are aggregated to form the weight of logical links, therefore the view that routers have of the network state is just an approximation of the real values that represent the state of each individual link.

Other sources of inaccuracy are the propagation delay of routing messages in large networks, the utilization of estimates about the current state of the network, and the impact of the metrics measurement mechanism used. Due to this wide range of factors, the global state that is kept by each router is just an approximation of the real actual state. When the path computation algorithms use this inaccurate information as if it was exact, their performance can be highly damaged, and thus solutions must be found to address this problem. Probabilistic approaches to address the inaccuracy in routing information aim at finding a path that is the most suitable to accommodate a new request, taking into consideration that the information available about the state of the network is inaccurate and represented by a probabilistic function [30] and [37]. Message probing is another technique used to deal with imprecise state information. The utilization of probing avoids the staleness of link-state information because the probes gather the most recent state information [32]. Multiple-path routing is able to reduce the impact that stale routing information has on routing performance, when compared to single path routing algorithms, due to its load balancing capability [8] and [30] and [38-43].

The problem of routing information inaccuracy is tightly related with the methods used for the distribution and aggregation of routing information, and thus new QoS Routing schemes to cope with this problem need to be developed in an integrated way, both at the algorithmic and protocol levels.

\subsubsection{QoS routing stability}

The stability of QoS Routing protocols is a determinant factor for their performance. Instability may occur whenever the responsiveness of the protocol becomes exaggerated, introducing thus unnecessary re-routing of traffic. Specifically, in link-state protocols, the inappropriate flooding of updates may originate route flaps that will degrade traffic performance. This is particularly problematic when the network is congested, since the additional routing messages consume the already scarce bandwidth resources, and the subsequent application of the path computation algorithm imposes even more load on the router processor.

The problem of routing instability is influenced by several factors, namely, the type of metrics used to compute the best path, the policy that controls the advertisement of the metrics and the path computing algorithm. Network topology and traffic patterns also influence routing behaviour and stability. Namely, shortest path routing based on a congestion based link metrics is very prone to instability under heavy loads and bursty traffic. The mechanisms to control the instability problem introduced above can be classified in three main categories, namely, concerning the metrics distribution mechanism, the path selection algorithms and the differential treatment of traffic with different QoS requirements.

The advertisement of quantified metrics is the first approach to avoid routing instability. The metrics quantification can be done using a simple average of the measured metrics [33] or using hysteresis mechanisms and thresholds [44]. Load-balancing techniques provide ways of utilizing multiple paths between a source and a destination, contributing to avoid routing oscillations [29] and [45]. Route-pinning [46] and class-pinning [47] are also used to limit routing oscillations is situations where the QoS paths are able to provide an adequate level of QoS. Stability and overall routing performance can be increased by using routing protocols that treat traffic flows according to their duration, and that perform route computations according to the mix of traffic, considering the individual needs of besteffort and QoS sensitive traffic [42] and [48].

The desired adaptability of QoS Routing schemes can not result in instability. However, these two objectives can be contradictory and the solutions for one can deny the satisfaction on the other. New QoS Routing algorithms should be designed such that routing oscillations are avoided, while providing for paths adequate to the type of traffic in the network.

\subsection{Multicast routing}

The multicast problem is that of routing from a single source node to a set of $p$ destination nodes, also called pointto-multipoint routing. The advances in technology and the fast emerging multimedia applications have provided great impetus for new (real-time) multicast applications. Many multicast applications (e.g., gaming, video-conferencing, audio and video streaming) will not operate properly if QoS cannot be guaranteed. Hence, future multicast algorithms must be capable of satisfying a set of QoS constraints.

A main property of multicast routing is the efficient use of resources. Because each of the $p$ destination nodes will receive the same information, sending the information $p$ times over each shortest path to each individual participant (i.e., unicast) is inefficient, since most likely there will be some overlap among the set of shortest paths. Multicasting as few duplicate packets as possible and only duplicating them if necessary is clearly more efficient. For the case of a single metric, multicast source routing can be implemented by forwarding the packet of a flow or session over a Steiner or shortest paths tree. However, a multicast tree may not always guarantee the requested QoS constraints, while multiple unicast QoS sessions can. This property enhances the complexity of constrained multicast routing (besides the proven NP-completeness), since we have to maintain a set of paths/trees and we need to check if no $\mathrm{min} / \mathrm{max}$ constraints are violated (merely topology filtering may be insufficient). A trade-off between efficient use of resources 
and QoS has to be made. The MAMCRA (Multicast Adaptive Multiple Constraints Routing Algorithm) algorithm [22] has recognized this trade-off and finds the set of shortest paths to all destinations and then reduces the consumption of resources without violating the QoS constraints.

We have indicated that guaranteeing QoS and optimizing resource utilization are two conflicting interests. Depending on the wishes of the client (multicast member), a trade-off can be made between QoS and resource utilization. This trade-off will be based on monetary cost, since guaranteeing a high level of QoS will inflict a large consumption of resources, which has to be paid for. It is not likely that all members are willing to pay the same price. Perhaps we can benefit from this user heterogeneity in QoS multicast routing. It would therefore be beneficial if some sort of negotiation between QoS and price could take place with the underlying objective to always strive towards a multicast tree.

The task of efficiently forwarding/replicating packets is part of the multicast protocol and not of the multicast algorithm. Several traditional multicast protocols exist, like DVMRP [49], MOSPF [27] and PIM [50] and some new QoS multicast protocols have been proposed. However, the area of multicast QoS Routing is still fairly unexplored (mainly because unicast QoS Routing is not fully understood yet as indicated by the previous sections) and hence much work can be done.

Finally, inspired by Connectionless Multicast (CLM), we touch upon Diffserv multicast and its exact active counterpart. In CLM, the packet header carries the IP addresses of all the multicast members. Each router determines the next hop for each destination and constructs a new header for every distinct hop. The new header only contains destinations for which the next hop is on the shortest path. In conformance to unicast Diffserv, we can extend CLM, such that each packet belongs to a certain Class of Service $(\mathrm{CoS})$ and each router has a routing table for each CoS.

Destination-based QoS Routing can only be guaranteed in an active network. If we store the history of an active packet in its header, then for each packet arriving at a router, MAMCRA could be used to compute the best forwardin$\mathrm{g} /$ replication strategy. The best use for such a CLM strategy is in highly dynamic (e.g. wireless) environments, since we do not need (to recalculate) routing tables. However, we do need to have an accurate view of the network. Some interesting research questions are:

- How efficient is multicast QoS Routing? Can we find theoretic bounds? Preliminary simulations suggest that the set of paths returned by MAMCRA approximate a tree.

- Can we improve MAMCRA?

- If we confine to multicast trees, what is the loss in QoS?
- Can we benefit from user QoS heterogeneity? Should we adopt QoS negotiation?

- What are the requirements of a new QoS multicast protocol?

- How to add/remove multicast members, while keeping the same level of QoS?

\subsection{Link-disjoint routing}

The problem of finding disjoint paths in a network has been given much attention in the literature due to its theoretical as well as practical significance to many applications, such as layout design of integrated circuits, survivable design of telecommunication networks and restorable/reliable routing. Paths between a given pair of source and destination nodes in a network are called linkdisjoint if they have no common (i.e., overlapping) links, and node-disjoint if, besides the source and destination nodes, they have no common nodes. With the development of optical networks and the deployment of MPLS or GMPLS networks, the problem of finding disjoint paths is receiving renewed interest as fast restoration after a network failure is crucial in such kind of networks. In robust communication networks, a connection usually consists of two link or node-disjoint paths: one active path and one backup path. A service flow will be redirected to the backup path if the active path fails. Load balancing, another important aspect for communication networks to avoid network congestion and to optimize network throughput, also requires disjoint paths to distribute flows. Robustness and load balancing are both aspects of Quality of Service (QoS) routing, among others.

In general a link-disjoint paths algorithm can be extended to a node-disjoint algorithm with the concept of node splitting, i.e. replacing one node with two nodes that are linked together via a link with zero-valued weights, and therefore we suggest focusing on (maximally) link-disjoint QoS Routing.

An intuitive method to determine two shortest linkdisjoint paths between a pair of source and destination nodes consists of two steps: the first step retrieves the shortest path between a given pair of nodes in a graph. The second step is to prune all the links of that path from the graph and to find the shortest path in the reduced graph. This method has at least two disadvantages: (a) provided that two link-disjoint paths exist, there is no guarantee that they will be found and (b) the second link-disjoint path may have a significantly larger length than the first shortest path. To surmount these disadvantages, other methods have been devised to find a pair of shortest link-disjoint paths with minimal total length. Unfortunately, as shown in [18], these techniques are not easily extended to link-disjoint QoS Routing, where the objective is to find two (maximally) link-disjoint paths that obey the constraints and that preferably have minimal total length. Kuipers and Van Mieghem [18] therefore proposed 
DIMCRA (link-Disjoint Multiple Constraints Routing Algorithm), which is shown to be better than the simple method of removing a path and finding the second disjoint path. However, room for research remains, as indicated by the following research questions:

- How efficient is link-disjoint QoS Routing? Can we find theoretic bounds?

- Can we improve DIMCRA?

- In which situation totally link-disjoint is not possible and we must switch to maximally linkdisjoint?

- Should we have the same constraints for the active as well as the backup path?

- Should we reserve the resources on the backup path?

- What is the best protection scheme?

- What are the requirements of a new QoS linkdisjoint protocol?

\subsection{The prediction-based routing approach}

The Prediction-based routing approach (PBR) has been already proposed as a precomputation scheme in optical transport networks [51]. An effort is being done to apply the PBR to traditional IP networks. There are many existing proposals dealing with precomputation or prediction issues, such as (i) the well known hot-potato routing [52] that 'predicts' the best route to a destination based on the information about the delay of requests that come from that destination, (ii) the proposal in [53] that predicts future traffic load in a link based on past measured samples of the traffic load in that link, and (iii) authors in [54] present a dynamic variation of the hot-potato routing. Contrary to these proposals, the PBR predicts links and routes availability instead of predicting incoming traffic load. Table 1 shows main differences among such proposals.

The PBR is based on the ideas of branch prediction in computer architecture [55]. In this area it is interesting to know if a branch instruction will be taken or not before computed in order to speed up the processor. By extending the concept of branch prediction to computer architecture, it will be necessary to register the history of the network state from the point of view of the source node, i.e. source nodes contains one register for every route from that node, which is updated with the occupancy information (bandwidth percentage of the total path capacity). It is worth noting that such registers are not updated by means of traditional update messages (including network state information), but at certain time cycles. The information obtained from the history registers is used to access the prediction tables. There is in fact on every source node one prediction table per feasible route from that source node. The prediction tables have different entries, each one keeping the information about a different pattern by means of a two bit counter. The prediction is done reading the value of such a two bit counter. A route is selected only if the value is 0 or 1 .

The algorithm used to select the paths is explained by means of an example. We suppose that between every source-destination pair there are two routes calculated, but the algorithm can be implemented for more than two routes. The history registration is only about the occupied bandwidth in the last cycle with 2 bits.

Fig. 2 represents the following example: when a new request demanding $40 \%$ of bandwidth reaches the source node the first route is examined. The last information about occupied bandwidth shows that a $40 \%$ is already used in this first route. Both bandwidth values are added $40 \%+40 \%$, and if it is less than $100 \%$ the prediction table of the first route is checked, otherwise the next prediction table would be checked. In this case the total bandwidth is $80 \%(>75 \%)$ and the index to access the first prediction table is $0(00$ coded in 2 bits). With this index the prediction table of the first path is accessed and the counter is read. Assuming that the counter is 2 , then the prediction is not to use this first route so that the second route will be examined. In this second path the occupied bandwidth in the last cycle was $25 \%$. The new bandwidth will be $40 \%+25 \%=65 \%$, which corresponds to an index of 1 (01 coded with 2 bits). With this index the prediction table of the second route is accessed and the counter value is 1 . This counter value means that the prediction is to use this second route hence the algorithm selects this second route.

As stated above, the history registers are updated every cycle with the information about the occupied bandwidth for the source node in every route. In the last example, when the algorithm selects the second route, the new bandwidth occupied by this node in this second route will be $65 \%$. It is important to note that this occupied bandwidth is only the bandwidth that the node knows, but it might not be the real occupancy. This situation occurs owing to remove update messages, since other source nodes might use more bandwidth in links of the same route and the source node

Table 1

Comparison of several precomputation algorithms

\begin{tabular}{|c|c|c|}
\hline Existing proposals & Advantages & Disadvantages \\
\hline Hot-potato routing & Learning Capability & No flow control \\
\hline Bandwidth estimation & $\begin{array}{l}\text { Learning Capability Accurate bandwidths esti- } \\
\text { mations }\end{array}$ & Update message are required \\
\hline Prediction-based routing & Learning Capability Without update messages & Learning from fails (from blocked requests) \\
\hline
\end{tabular}






Fig. 2. Example of prediction with two routes.

occupancy information will not be updated. Only the prediction table of the selected route is updated. If the connection is set-up the corresponding counter of the prediction table is decreased, but if the connection is blocked the counter is increased. In the above example if the connection is established the counter of the entry 01 of the prediction table of the route 2 will be 0 , but if the connection is blocked the counter will be 2 .

The following aspects are still open in the PBR mechanism:

- How many bits are needed to register the occupied bandwidth of the last cycle?

- How many previous cycles are needed to register?

- If there is information about the last and previous cycles, how is this information hashed to build an index to access the prediction tables?

- What happens when the algorithm does not select any route because it predicts that all are occupied?

\section{Inter-domain issues}

The Border Gateway Protocol (BGP) is currently the defacto standard inter-domain routing protocol in the Internet. Its current release is BGP-4, which was specified in [28] on March of 1995. Throughout these years the number of Autonomous Systems (ASs) connected to the Internet has augmented enormously, which accordingly increased the demands on the scale of the network. In spite of this burden, BGP has proven to be a resilient routing protocol. Among the strengths that made BGP become so popular are firstly that it was designed to address the issues of scalability and connectivity demands at a very large scale. Secondly, it has demonstrated to be able to provide adequate stability to the biggest network ever deployed, and thirdly, it was endowed with policy based routing features allowing each administrative domain at the edge of a BGP connection to manage its inbound and outbound traffic according to its specific preferences and needs. Finally, it is worth noting that BGP has very flexible mechanisms which allow easy developments and extensions to the protocol (e.g. BGP communities attribute and multiprotocol extensions for BGP).

Despite these significant strengths, BGP also presents several weaknesses. For instance, in many cases BGP requires tens of minutes to recover from a route or a link failure [56]. Moreover, even though BGP allows an AS to flexibly manage its outbound traffic, it exhibits a scarce degree of control in order to manage and balance how traffic enters an AS across multiple possible paths. In addition, each BGP router only advertises the best route it knows to any given destination prefix. This implies that many alternative paths that could have been potentially used by any source of traffic will be unknown because of this pruning behavior inherent to BGP. The justification for this behaviour is that BGP was intrinsically designed to address overall stability and scalability instead of concerning about issues like fast recovering from a particular link failure, nor bounding delay or the packet loss ratio across the Internet for a given block of prefixes, just to name a few. In summary, the current release of BGP supplies a slow reacting and limited routing protocol, which is inadequate to handle most of the emerging demands for inter-domain functionalities. Among these inter-domain demands is the absence of highly efficient and cost-effective mechanisms to supply different levels of end-to-end Quality of Service $(\mathrm{QoS})$, in which the inter-domain routing protocol is of utter importance. In other words, the current release of BGP lacks of QoS Routing capabilities which has been already recognized as a strong need by the Internet Engineering Task Force (IETF) since mid-1998 [3]. Consequently, several efforts are being carried out to address the issue of QoS Routing at an inter-domain level in IP networks.

\subsection{QoS extensions and traffic engineering using BGP}

Many researchers and manufacturers are trying to enhance BGP with new capabilities such as Traffic 
Engineering, and QoS extensions, mainly because of the ubiquity and success that BGP presents at this moment. Proposals such as [57-61] are very good examples of this kind of approach to the issue. It is important to notice that even though the non-extended version of BGP presents limited functionality, it is indeed a complex routing protocol, where mistakes and misconfigurations are not infrequent. Moreover, some research groups have extended BGP with layer 2 and layer 3 Virtual Private Network (VPN) discovery and signalling capabilities, within the new Multi-Protocol BGP (MP-BGP). As a result, these proposals for enhancing BGP not only tend to turn it into a much more complex protocol, but also it remains to be seen if the addition of all these enhancements in a real environment could not overwhelm the protocol.

\subsection{Overlay approaches}

Rather than enhancing BGP an alternative to interdomain QoS Routing is the overlay approach, which has become a strong candidate to address the issue. Proposals such as [62-66] reflect this kind of approach. The main idea behind the overlay concept is to decouple part of the policy control portion of the routing process from BGP devices. In this sense, the two approaches differ in how policies are controlled and signalled. BGP enhancements tend to provide in-band signalling, while the overlay approach provides out-of-band signalling. In any case, it is important to keep in mind that at present the only way to engineer inter-domain traffic in IP networks is by means of smartly configuring BGP, so at the end both mechanisms rely on appropriately tuning BGP to comply with their respective traffic policies. It is worth noticing that while the former approach provides significant improvements for internets under low routing dynamics, the latter is more effective when routing changes occur more frequently. From our perspective, whereas significant extensions and enhancements to BGP are certainly going to be seen, the overlay structure arises as a strong candidate to provide flexible and value-added out-of-band inter-domain QoS Routing. In particular, this becomes perfectly suitable when interdomain traffic patterns need to dynamically adapt and rapidly react to medium or high network changing conditions, where the former solutions seem impracticable at the present time.

The Overlay Architecture is mostly appropriate when communicating domains are multihomed, and thus may need some kind of mechanism to rapidly change their traffic behaviour depending on network conditions. Multihoming is the trend that most stub ASs exhibit in nowadays Internet, which mainly try to achieve load balancing and fault tolerance on the connection to the network. As a matter of fact, at present nearly $80 \%$ of the more than 17,000 Autonomous Systems (AS) that compose the Internet are stub AS, where the majority of this fraction is multihomed. In addition, present inter-domain traffic characteristics reveal that even though an AS will exchange traffic with most of the Internet, only a small number of ASs is responsible for a large fraction of the existing traffic. Moreover, this traffic is mainly exchanged among ASs that are not directly connected; instead they are generally 2,3 and 4 hops away [60]. Thus, it is possible to conceive a completely distributed overlay architecture and routing layer specifically designed to provide inter-domain QoS Routing among strategically selected non-peering multihomed ASs. The foremost motivation for influencing traffic in this way is that with only a very small number of Overlay Entities (OEs), but located at strategically selected remote multihomed ASs is enough to control a significant part of the traffic for the most widely deployed kind of AS in the current Internet [66]. A major advantage of this framework is that no OEs are needed in any transit AS connecting the remote ASs in the overlay model. Thus, the complexity of dynamic QoS provisioning is pushed to the edge of the network by means of a distributed overlay architecture. In this scheme a pair of OEs within two remote multihomed ASs are able to exchange Service Level Agreements (SLAs) regarding the traffic among them, examine the compliance with those SLAs, and accurately configure on-the-fly the underlying BGP layer to bypass network problems such as link failures, or service degradation for any given Class of Service $(\mathrm{CoS})$. The essence in this approach is that the QoS perception between a pair of remote ASs is basically the one that the OEs have of each other.

The complexity of inter-domain QoS Routing increases significantly when compared with the problem of intradomain QoS Routing mainly because stringent end-to-end QoS demands for inter-domain resource reservation capabilities. In [8] the authors offer an interesting in-band solution to this issue. Alternatively, it is possible to conceive dynamic end-to-end inter-domain QoS without any kind of resource reservation, and to follow the IP connectionless paradigm, as long as only soft end-to-end QoS is guaranteed. Once again, the overlay approach arises as the perfect candidate to offer this kind of solution [65] and [66].

A major challenge in the overlay approach is how to provide a highly efficient coupling between the underlying BGP routing layer and the overlay routing layer. Furthermore, an attractive approach to inter-domain QoS Routing is to supply a complementary solution to the issue in which a completely distributed overlay architecture and a routing layer is used for dynamic QoS provisioning, while QoS extensions and/or TE capabilities of the underlying BGP layer are used for static QoS provisioning. In this sense, the overlay structure feeds from and reuses the best ongoing efforts in the area of in-band inter-domain QoS Routing for low dynamic QoS and/or TE provisioning [66]. Thus, in terms of the underlying inter-domain routing structure two types of BGP routers can operate, namely, non-QoS aware $\mathrm{BGP}$ routers and QoS aware BGP (QBGP) routers, where in order to develop highly scalable and stable routing schemes it is mandatory that QBGP routers only distribute non 
dynamic QoS information. This is mainly because frequent network changes will translate into frequent BGP updates, which may lead to routing instability. The reactive nature of the overlay routing layer acts then as a complementary layer conceived to enhance the performance of the underlying BGP layer containing both QoS and non-QoS aware routers. The distributed overlay approach to inter-domain QoS Routing offers several research challenges. Among the main unsolved issues are:

- Provide enough evidence that the compound routing model presents better performance than each of its parts separately.

- Provide enough evidence that a completely distributed overlay routing layer presents better performance than other more complex overlay architectures.

- An in-depth analysis of the scalability of the overlay approach

- Highly efficient coupling between the overlay and the QBGP/TE-BGP routing layers.

- Definition of supported QoS parameters, definition of supported CoSs and the set of negotiable SLAs.

- Development of secure overlay protocols, which will mainly handle the SLAs for different classes of services (network bundles), feedback (if necessary), and triggered routing updates.

- Definition of new QoS Routing algorithms where routes are selected based on novel metrics.

- Development of non-oscillating algorithms with the aim of maximizing the utilization of available network resources, but fulfilling the SLA constraints.

- Development of novel QoS Routing algorithms which avoid best-effort traffic starvation.

- Development of highly scalable and efficient monitoring and probing techniques, in order to be able to take accurate and rapid routing decisions constrained by the SLAs.

- Provide tentative solutions to the problem of autodiscovery of OEs.

\subsection{Multihoming}

Recent studies show that the sustained growth of the Internet routing tables, despite the explosion of the technology bubble and the consolidation in the Internet Service Provider markets, comes from medium size and larger corporations which need presence in the Internet. These corporations have their own autonomous system identifier (ASid) and an address range from the provider independent addressing space. They connect to two or more providers to achieve resilience in their access to the Internet.
A positive side effect of this strategy is that Internet Access Provider dependency is avoided.

One of the main goals of IPv6 was to provide tight aggregation of the address space for the routing core, in order to optimise the routing process in the core and keep the size of the routing table manageable. Furthermore, it is worth noting that practical deployment of IPv6 networks is showing a high level of reuse of technologies, techniques and best practices (for example, the exterior gateway protocol in the IPv6 Internet is BGP4+, which is an extension of BGP-4, the exterior gateway protocol of the IPv4 Internet).

But if multihoming (central issue for the success of a future Internet based on IPv6), as known in the IPv4 Internet is also applied in IPv6, the effect on the IPv6 routing table size is foreseeable. With a vastly greater addressing space, the size of the routing tables in the core of an IPv6 router with uncontrolled multihoming is likely to explode beyond manageability, or at least beyond the levels where efficient and cost effective core switching devices can be produced. More efforts must be devoted to propose a new approach to IPv6 multihoming. Consensus between providers and customers building around a solution which is both technologically sound and commercially viable is critical. This reflects in a complex development cycle for a complete solution.

Given the importance of the issue at stake-i.e. the stability and viability of the future IPv6 Internet-the current status of standardisation for IPv6 multihoming is not very encouraging. Multihoming in IPv6 has been viewed as a problem of the end host, suffering under the fact that the IPv6 standards allow multiple IPv6 addresses to be assigned to end terminals. There are two RFCs studying the general goals of multihoming at site level: [67] presents the goals and [68] proposes a partial solution to allow multihoming support at the site exit. Ambiguities at end system or site level have been ignored up to the moment.

An initiative to integrate DNS information in the routing process at host level, allowing the end system to select the source IP address in an interface with multiple IP addresses [69], has not found enough response.

Multihoming at a provider level has not generated much interest either, and some proposals to structure the connection of ISPs [70] have not generated enough interest and thus, have never been promoted from Internet Draft to RFC. Multihoming at ISP level is a common practice in today's Internet. Two main flavours of BGP-4 supported multi-homing are being used. Small companies buying their Internet connectivity from one provider might be connected via two or more independent links, in order to enhance reliability. In this case, the client uses a private Autonomous System identifier in its peering with the provider. In the case where the addressing range assigned to the client is not aggregated into one of the provider's addressing range, this identifier is removed at the provider's peering points and the client's addressing range appears as one of the provider's 
addressing ranges. When multihoming to several providers, the multihoming client needs a public autonomous system identifier, which progresses through all the BGP-4 peering to the Internet's routing tables.

RIPE's Routing Information Service (RIS) [71] has over 300 IPv4 and IPv6 peers at 12 data collection points worldwide, which collect and store Border Gateway Protocol (BGP) routing information and make it publicly available for the Internet community. A large archive of raw RIS data dating back to September 1999 is available for research purposes. This RIS project publishes a set of tools to harvest and analyse the Route Repositories.

The RIS database manages a huge amount of routing data and the mixed IPv4/IPv6 nature of the current repositories creates a huge overhead when processing them. This can be remedied by establishing new, IPv6 specific Route Repositories on pure IPv6 infrastructures. Candidate deployment environments for these new routing repositories are the different IST projects in the 6th Framework Programme [72], which have implemented native IPv6 networking infrastructures as well as national research networks, which have deployed or are deploying pure IPv6 networking infrastructures.

In the scope of serious IPv6 multihoming studies, the RIS toolset needs to be enhanced with specialised tools, which study the effect of multihoming practises in emerging IPv6 networks. The study of current multihoming practises and their impact on the current Internet should only be considered as a starting point.

IPv6 multihoming solutions following the current IPv4 practices will render the IPv6 Internet not viable at some point in time. In order to improve the situation, multihoming awareness has to be introduced in the protocol level. Since BGP-4 has multiprotocol capabilities-which, i.e. make BGP-4 routing in IPv6 possible-the main work is defining the mechanisms controlling multihoming.

The Route Repositories are very useful in the design process of the BGP4 + multihoming extensions. They will provide real world data, on which the proposed multihoming extensions to BGP4+ can be simulated. A debugged prototype implementation of the proposed multihoming standard can be deployed in a testbed network. After validation, the protocol should be implemented and deployed on selected routers of a production IPv6 network.

BGP-4 as a routing protocol has a flaw in its design. While other routing protocols have a clearly defined objective, i.e. find the route between two points which is optimal under a well defined set of criteria, BGP-4's main objective is to provide routing continuity between autonomous routing domains. The policy independence between autonomous systems and the opacity of routing policies outside the autonomous system lead to instabilities. Research on the inconsistencies of BGP-4 has led to the discovery of situations, where the coordinated action of the administrators of more than two routing domains is needed to restore stability in a certain subsection of the Internet [73] and [74]. A routing management overlay, which could be able to detect and avoid this kind of situations would greatly improve the quality of the IPv6 Internet. Such an overlay will have detractors, which will argue the autonomy of the Autonomous System is put in jeopardy by such an approach. A paradigm shift is needed and some current practises have to be modified. Competing but collaborating Internet Service Providers will be able to achieve better service levels than isolated Internet Providers.

\section{Optimization issues}

As reminded in the introduction, an algorithm solving the MCOP problem searches for the smallest length path within the set of feasible paths. Each of such feasible paths obeys the QoS constraints and, therefore, satisfies user's requirements. The path length function is thus a degree of freedom we can exploit to meet service provider's perspective, too. The feasible path optimizing network resources should be selected. If there exists only one feasible path, the algorithm has no choice: the unique feasible path must be assigned to the flow (we are implicitly assuming a greedy admission control). But, if multiple feasible paths are available, the algorithm has to select one of them. Choosing one feasible path rather than another leads to a different occupation of resources. The network status (in terms of link utilization) seen by future flows depends on which feasible path is chosen. Therefore, such a choice has eventually an impact on blocking of requests and network throughput.

Traffic engineering algorithms [75-78] have been evaluated from the viewpoint of resource optimization in a dynamic scenario. They focus on the definition of a proper link cost function (the length of a path being the sum of the costs of its links), disregarding additive QoS constraints. Thus, they can simply make use of the Dijkstra's algorithm to find the optimal path. Some of the authors claim that additive QoS constraints can be dealt with by converting them into an effective bandwidth requirement. Thus, if it is possible to reserve an amount of bandwidth equal to the computed effective bandwidth along the path, all the QoS constraints are satisfied. We believe that such an approach is effective only if routers are able to reserve the requested bandwidth for each flow. Integrated Services (IntServ) is an architecture based on this concept, but this per-flow QoS is paid for with complexity and lack of scalability. In a Diffserv (Differentiated Services) network, instead, flows requiring the same treatment are aggregated into a single macroflow. We first state our claim and then illustrate it by means of an example.

Proposition 1. In a network with routers arranged for perclass scheduling (e.g. Diffserv), if the routing algorithm converts additive QoS constraints into an effective bandwidth requirement and selects the path based only on such requirement, then the QoS granted to already admitted flows may not be preserved after new flows are routed. 
Assume that at a certain point all the flows inside the network receive the requested treatment, that is for each flow $\sum_{l \in P} q_{i}(l) \leq L_{i}, i=1, \mathrm{~K}, m$, where $P$ is the path selected for the flow and $q_{i}(l)$ is the average value of the $i$-th additive QoS metric a packet of that flow experiences crossing link $l$. Since there is no differentiation among the packets of flows belonging to the same service class, $q_{i}(l)$ is the same for all the flows of a class. As an example, Fig. 3 (a) depicts such a situation for $m=2$. The two-component vector shown around each link represents the pair $\left[q_{1}(l)\right.$, $\left.q_{2}(l)\right]$, the average values of the two additive QoS metrics perceived by each packet crossing the link. Among the flows already routed, consider flow 1 that requires a QoS level represented by the constraint vector $L=[12,10]$ and has been routed along the path $P_{\mathrm{ABEF}}$. The QoS constraints are clearly satisfied since $2+4+5=11<12$ and $3+3+1=$ $7<10$. When a new flow arrives, its effective bandwidth is computed and, provided that there are enough resources, it is routed along a certain path. Suppose flow 2 (requiring a QoS constraint vector $L=[13,14]$ ) is admitted and routed along the path $\mathrm{P}_{\mathrm{ABCF}}$. Routing this new flow causes $q_{i}(l)$ to deteriorate for $l$ belonging to the path $\mathrm{P}_{\mathrm{ABCF}}$. In particular, the QoS across link $A \rightarrow B$ becomes, for example, [4,4] (Fig. 3 (b). This makes the QoS constraints of flow 1 no longer satisfied, since $4+4+5=13>12$ and $4+3+1=$ $8<10$.

Therefore, our insight is that routing algorithms should explicitly take into account additive QoS constraints. On the other hand, most QoS Routing algorithms typically assume a frozen view of the network and the focus of their authors is on complexity issues rather than on dynamic behaviour optimization. Such gap may be filled by defining a proper length function. SAMCRA is an exact QoS Routing algorithm that can be used with any path length function. The length of a path $P$ proposed in [5] is shown in (2).

$$
l(P)=\max _{1 \leq i \leq m} \frac{w_{i}(P)}{L_{i}}
$$

SAMCRA further makes use of a $k$-shortest path approach, which is essentially a version of the Dijkstra's algorithm that does not stop when the destination is reached, but continues until the destination has been reached $k$ times. Not all sub-paths are stored, but an efficient distinction based on non-dominance is made. A (sub-) path $P_{1}$ is said to be dominated by a (sub-)path $P_{2}$ if $w_{i}\left(P_{2}\right) \leq w_{i}\left(P_{1}\right)$ for $i=$ $1, \ldots, m$. SAMCRA only considers non dominated (sub-) paths. The non-dominance check guarantees that the dominated sub-path does not lead to a feasible path if the dominant sub-path does not lead to a feasible path. Also, it assures that the dominated sub-path does not lead to a path with a length smaller than that of the path the dominant subpath leads to. The path length $l($.) can also be a function of the available bandwidth of the links of the path. In such a case the non-dominance check needs to include the additional bandwidth parameter.

Thus, it is possible to use an exact QoS Routing algorithm with a suitable path length function to optimize network dynamics. But, which path length function has to be used is still an open issue. A first conceptual difficulty resides in the definition of the optimum criterion. A service provider might be interested in having the traffic load fairly distributed among network links. Or, (s)he might be interested in maximizing the throughput and the rate of accepted user requests. It is not proven that one of these objectives implies the other or vice versa. They might even be contrasting. Another difficulty arises when we try to formalize the chosen optimum criterion. To pursue the objective of optimizing network resources, we can exploit the freedom of choosing a path length function. But, which is the path length function that enables to achieve a specified goal? The state-of-the-art research on this topic is not able to provide an answer. Neither are there attempts to organize the problem in a mathematical framework. Most of the works in the literature propose heuristic path length functions. The performance of the proposed algorithms is typically evaluated through simulations. Each work considers its own network topologies, link capacity distributions and traffic loads. The network is filled with a number of user requests and statistics related to various performance indices are collected. From such analysis it is

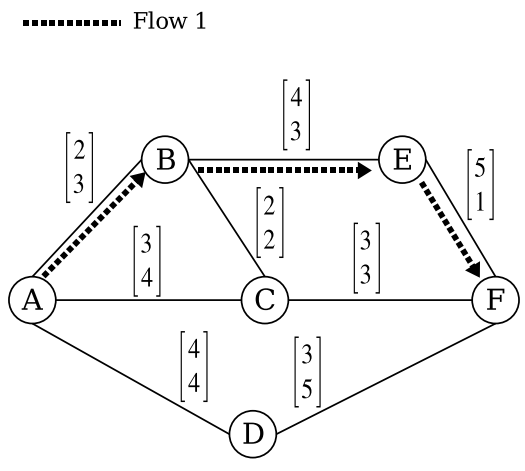

(a)

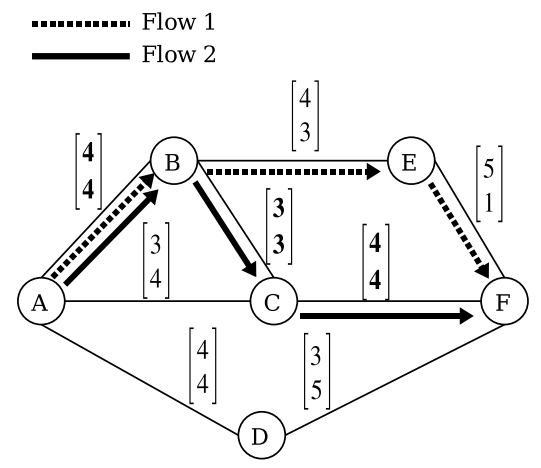

(b)

Fig. 3. Sample example to illustrate Proposition 1. 
not possible to draw general conclusions, but only infer the behaviour in a specific scenario. A complete understanding of network dynamics is therefore still missing.

\section{Cross-Layer QoS routing on wireless networks}

Routing in wireless networking organizations is challenging, since the radio environment may be hostile and often unstable, introducing new performance issues. Moreover, wireless networks such as ad-hoc or Wi-Fi were firstly designed with a clear separation of layer's functionalities, neglecting the important characteristics of their physical and link layers. It has been proved that routing in multi-hop wireless networks using the traditional Shortest-Path metric is not a sufficient condition to construct optimal paths able to effectively transport data with reasonable delay, throughput and reliability. Indeed, the shortest-path metric approach does not take into account the variable quality of the wireless link. Other solutions, i.e. other QoS metrics, that are aware of the wireless nature of the underling physic medium of the system, should be explored. In this context, QoS Routing becomes fundamental but also a harder problem to solve. Indeed, as mentioned in Section 1.1, we are still on the way to have a complete conceptual understanding and theory of QoS Routing. This is true in wired environment, which is a consolidated technology, while wireless multi-hop environment is a relatively new technology, whose issues are far from being completely explored, adding more and new issues to QoS Routing.

To give at routing level the awareness of the underlying wireless channel, a cross-layer approach is unavoidable. Nevertheless, a general framework for cross-layer approach has not yet been defined. This falls in the dynamics aspects of QoS Routing. The parameters the cross-layer approach should make available at routing level are equivalent to the links weight. Thus most of the issues listed in Section 1.3, should be considered in defining a cross-layer framework. Further, the parameters (weight) that are sampled from the lower layers must have a simple additive rule, in order to maintain a low level of complexity in calculating paths.

Routing performed at the Network Layer, having knowledge of the whole network, achieves better performance when considering the wireless nature of the underlying medium. The goal is to find paths that can be used by real applications, and thus that offer some minimal QoS, while considering that each transmission is going to lower QoS of other nodes. This leads to the general problem of QoS Routing, on which plenty of work has already been published, based on wired networks. In the wireless multihop scenario, the Routing Layer should be fed with metrics sampled at lower level and with an overall abstract view of the MAC and PHY layers. This lower layer sampling, coupled with the global view of the Network Layer permits to implement heuristics targeting main significant factors limiting global network performance, such as
Interference and link's QoS, based on several different parameters (i.e. metrics) that characterize the wireless medium. At the same time, since link's quality may change quickly, the routing algorithm should be able to damp oscillating effects of the wireless environment. The Cross-Layer approach, integrating the interaction of the different layers, is useful in order to incorporate the physic of the complete system. In short, cross-layer routing in wireless multi-hop networks can be also formalized as a $\mathrm{MC}(\mathrm{O}) \mathrm{P}$ problem, which is NPComplete. Algorithms and heuristics already available for NP-Complete problems can be adapted to the wireless case. Wireless multi-hop networks can be classified in three main categories:

- Sensor Networks

- Ad-Hoc Networks

- Mesh-Networks

While, on the one hand all of them are based on the multi-hop technology, on the other hand they have different goals, architecture, thus different QoS requirements. Crosslayer QoS Routing may lead to great improvements in all the three categories.

Sensor networks are difficult to put in a framework, since each implementation strongly depends on the application. Nevertheless, reliability, timeliness and survivability are the most important qualities that should be satisfied. These are not usual QoS constrains, like bandwidth, or delay, but routing has to take into account some requirements that the specific platform wishes to fulfill. Supporting reliability at routing level, avoiding retransmissions at both MAC and application level, improves the timeliness. This gives another example of the fundamental concept of dependability, described in Section 1.5.

Ad-Hoc networks are a mobile self-organizing flat architecture. Since each user can move freely, link quality changes are faster and more unpredictable. Lifetime of paths is strongly reduced and QoS Routing should be able to readily find new paths, in order to maintain connectivity, while preserving QoS requirements. Complexity becomes a critical issue and has to be reduced. Indeed, if the algorithm complexity is high, the risk is that the algorithm is not able to find a usable path, spending all the time chasing changes that happen in the network. Optimality may be sacrificed in order to reduce the computational time, using a heuristic approach. Moreover, a higher computational load leads to a higher energy consumption that may reduce lifetime of the user's device.

Wireless Mesh Networks appear as a promising technology to offer broadband wireless access to the Internet, but also to build self-organized networks in places where wired infrastructure is not available or not worth to deploy. Mesh architecture is based on wireless routers that are able to self-configure themselves as an access or a backbone network, offering connectivity to 
end-users by means of standard radio interfaces. If one or more wireless routers have a wired connection to the Internet, they also act as gateways, relieving the need to ensure one Internet connection per access point as in standard solutions.

Mesh router have a slow degree of mobility, it is more likely that they are fixed while present in the network, however, their number may change dynamically to adapt the capacity of the network to traffic conditions or number of final users. In this context, algorithm's complexity is less critical than in ad-hoc networks. Mesh routers can give more focus to optimality in order to find best paths toward each router, while respecting constrains.

A wireless multi-hop cloud can also be viewed as a network domain. This is not unrealistic, since the actual vision of the future Internet consists on an optical switched core and wireless multi-hop technology at the edges. As a network domain, wireless multi-hop networks share some issues with intra-domain QoS Routing (see Section 2). At the same time, since wireless clouds may communicate one to another, some inter-domain issues arise (see Section 3).

Main research challenges in wireless multi-hop QoS Routing:

- Prototype cross-layer approach to enable QoS multi-hop routing.

- Trade-off between QoS algorithm complexity and rapid changing conditions, like channel instability and user mobility.

- Development of algorithms and metrics that damp oscillating effects of wireless environment.

- The choice of the link weights has a deep impact on the stability of the routing solution. New metrics should be explored, but attention should be paid to the addition rule.

- In multi-hop environment routing choices have deep impact on the quality of the links (e.g. interference). Decision constrains should take it into account.

\section{Focus of partners within E-Next}

The E-Next (Emerging Networking Experiments and Technologies) Network of Excellence funded by the Framework Programme 6, targets to drive Europe to become a word leader in a key area of Information Society Technologies, namely computer networking. This goal can be achieved by developing a critical mass of expertise made of both the best people and labs doing research on computer networks. Many of the people integrating E-Next is jointly collaborating in topics closely related to QoS innovation and deployment. Thus, the focus of the partners in E-Next in the field of QoS
Table 2

Main interests of the E-Next partners involved in this article

\begin{tabular}{ll}
\hline E-Next partner & Devoting efforts in \\
\hline UPC & Algorithm, dynamics, intra/inter-domain routing \\
UoC & Algorithm, dynamics, intra/inter-domain routing \\
TUDelft & Algorithm, dynamics, multicast, link-disjoint \\
Federico II & Optimization issues \\
Telefonica & Multihoming \\
TUD & Routing dependability, mobile and wireless networks \\
LIP6/CNRS & Wireless networks \\
\hline
\end{tabular}

Routing (as set forth in this article) is that shown in Table 2.

\section{Conclusions}

In this paper we have surveyed many unresolved research challenges in QoS Routing showing that while several algorithmic aspects of QoS Routing still need to be addressed, the majority of those challenges lie in its dynamic aspects due to its remarkable complexity.

We have also independently analyzed the most important open issues in the areas of intra-domain and inter-domain routing, presenting at the same time some of the most compelling proposals and ongoing research efforts in both routing areas. In addition, optimization issues and the most important research challenges in wireless multi-hop QoS Routing were presented. In summary, our aim in this paper was basically to:

- Support the necessity of feasible and costeffective QoS-aware routing protocols that could be able to cope with the requirements and functionalities that most of the emerging network services impose

- Clearly expose the most important open issues in the area of QoS Routing

- Briefly present an up-to-date set of proposals that address some of the challenges in QoS Routing, and also highlight some promising ongoing research efforts done both inside and outside the E-Next community.

Besides the overview of current research work on QoS routing in the scope of the E-NEXT Network of Excellence we believe that this paper can contribute to the identification of open research issues in the field and to attract other researches to this important research field.

\section{Acknowledgements}

This work has been supported by the European Commission within the IST E-Next project FP6-506869 


\section{References}

[1] Telecommunication Standardization Sector of ITU. ITU-T Recommendation E.800: Terms and Definitions related to Quality of Service and Network Performance including Dependability, 1994.

[2] P. Van Mieghem (ed.), F.A. Kuipers, T. Korkmaz, M. Krunz, M. Curado, E. Monteiro, X. Masip-Bruin, J. Solé-Pareta, and S. SánchezLópez, Quality of Service Routing, Chapter 3 in Quality of Future Internet Services, EU-COST 263 Final Report, edited by Smirnov et al. in Springer LNCS 2856, pp. 80-117, 2003.

[3] Z. Wang, J. Crowcroft, Quality of service routing for supporting multimedia applications, IEEEJSAC 14 (7) (1996).

[4] H. De Neve, P. Van Mieghem, TAMCRA: a tunable accuracy multiple constraints routing algorithm, Computer Communications 23 (2002).

[5] P. Van Mieghem, F.A. Kuipers, Concepts of exact quality of service algorithms, IEEE/ACM Transaction on Networking 12 (5) (2004) 851-864.

[6] G. Apostolopoulos, R. Guerin, S. Kamat, S. Tripathi, Quality of service based routing: a performance perspective, Proceedings of SIGCOMM'98, Vancouver, British Columbia, USA (1998).

[7] R. Guérin, A. Orda, Computing Shortest Paths for Any Number of Hops, IEEE/ACM Transactions on Networking 10 (5) (2002) October.

[8] Y. Jia, Y. Nikolaidis, P. Gburzynski, Alternative Paths vs. inaccurate link state information in realistic network topologies, Proceedings of SPECTS'02, San Diego, CA (2002) 162-169.

[9] Q. Ma, P. Steenkiste, Routing traffic with quality-of-service guarantees in integrated services networks, Proceedings of Workshop on Network and Operating Systems Support for Digital Audio and Video (1998).

[10] J.L. Sobrinho, Algebra and algorithms for QoS path computation and hop-by-hop routing in the Internet, IEEE Transactions on Networking (2002).

[11] G. Cheng, N. Ansari, Finding all hops k-shortest paths, Proceedings of 2003 IEEE Pacific Rim Conference on Communications, Computers and Signal Processing (PACRIM'03), Victoria, BC, Canada (2003) 474-477.

[12] F.A. Kuipers, T. Korkmaz, M. Krunz, P. Van Mieghem, An overview of constraint-based path selection algorithms for QoS routing, IEEE Communications Magazine 40 (12) (2002) December.

[13] H. Salama, D. Reeves, Y. Viniotis, A distributed algorithm for delayconstrained unicast routing, INFOCOM'97, Japan (1997).

[14] M. Curado, J. Brito, B. Melo, G. Quadros, E. Monteiro, Quality of service routing in the differentiated services framework, Proceedings of SPIE's International Symposium on Voice, Video, and Data Communications, Boston, MA, USA (2000).

[15] Jüttner, B. Szviatovszki, I. Mécs, Z. Rajkó, Lagrange relaxation based method for the QoS routing problem, IEEE INFOCOM 2001, Anchorage, Alaska (2001).

[16] T. Korkmaz, M. Krunz, S. Tragoudas, An efficient algorithm for finding a path subject to two additive constraints, Joint International Conference on Measurement and Modeling of Computer Systems, Proceedings of the 2000 ACM SIGMETRICS international conference on Measurement and modeling of computer systems table of contents, Santa Clara, California, United States (2000) 318-327.

[17] F.A. Kuipers, P. Van Mieghem, The impact of correlated link weights on QoS routing, Proceedings. of IEEE INFOCOM 2003, San Francisco, USA (2003).

[18] Y. Guo, F.A. Kuipers, P. Van Mieghem, A link-disjoint paths algorithm for reliable QoS routing, International Journal of Communication Systems 16 (9) (2003) 779-798.

[19] F.A. Kuipers, T. Korkmaz, M. Krunz, P. Van Mieghem, Performance evaluation of constraint-based path selection algorithms, IEEE Network 18 (5) (2004) 16-23.
[20] F.A. Kuipers. P. Van Mieghem, 'Non-Dominance in QoS Routing: an Implementational Perspective', to appear in IEEE Communications Letters.

[21] F.A. Kuipers, Quality of Service Routing in the Internet: Theory, Complexity and Algorithms, Delft University Press, The Netherlands, 2004, ISBN: 90-407-2523-3.

[22] F.A. Kuipers, P. Van Mieghem, MAMCRA: a constrained-based multicast routing algorithm, Computer Communications 25 (8) (2002) 801-810.

[23] Avizienis, J.-C. Laprie, B. Randell, C. Landwehr, Basic concepts and taxonomy of dependable and secure computing, IEEE Transactions on Dependable and Secure Computing 1 (1) (2004) 11-33.

[24] Institute of Electrical and Electronics Engineers. IEEE CS Technical Committee on Fault Tolerant Computing and International Federation for Information Processing. IFIP WG 10.4 on Dependable Computing and Fault Tolerance. Available at http://www.dependability.org/.

[25] R. Perlman, Interconnections: Bridges, Routers, Switches, and Internetworking Protocols, second ed., Addison-Wesley, Boston, 1999.

[26] M. Hollick, I. Martinovic, T. Rimac, A survey on dependable routing in sensor networks, Ad hoc networks, and cellular networks 2004 pp. 495-502.

[27] J.Moy, 'OSPF Version 2', RFC 2328, 1998.

[28] Y. Rekhter, T. Li, A border gateway protocol 4 (BGP-4), Internet Engineering Task Force, RFC 1771 (1995).

[29] M. Curado, O. Reis, J. Brito, G. Quadros, E. Monteiro, Stability and scalability issues in Hop-By-Hop class-based routing, Proceedings of the 2nd International Workshop on QoS in Multiservice IP Networks (QoS-IP 2003), Milano, Italy (2003).

[30] G. Apostolopoulos, D. Williams, S. Kamat, R. Guérin, A. Orda, T. Przygienda, 'QoS Routing Mechanisms and OSPF Extensions', Internet Engineering Task Force, Request for Comments 2676, 1999.

[31] G. Apostolopoulos, R. Guerin, S. Kamat, A. Orda, S. Tripathi, 'IntraDomain QoS Routing in IP Networks: A Feasibility and Cost/Benefit Analysis', Special Issue of IEEE Networks on Integrated and Differentiated Services for the Internet, 1999.

[32] M. Claypool, G. Kannan, Selective flooding for improved quality-ofservice routing, Proceedings of SPIE Quality of Service over NextGeneration Data Networks, Denver, Colorado, USA (2001).

[33] Khanna, J. Zinky, The revised ARPANET routing metric, Proceedings of SIGCOMM'89, Austin, Texas, USA (1989).

[34] T. Korkmaz, M. Krunz, Source-oriented topology aggregation with multiple QoS parameters in hierarchical networks, ACM Transactions on Modeling and Computer Simulation (TOMACS) 10 (4) (2000) 295-325.

[35] F. Hao, E.W. Zegura, On scalable QoS routing: performance evaluation of topology aggregation, Proceedings IEEE INFOCOM 2000, Tel Aviv, Israel 1 (2000) 147-156.

[36] K. Lui, K. Nahrstedt, S. Chen, Routing with topology aggregation in delay-bandwidth sensitive networks, IEEE/ACM Transactions on Networking (TON) 12 (1) (2004) 17-29.

[37] Y. Goto, M. Ohta, K. Araki, Path QoS collection for stable Hop-byHop QoS routing, Proceedings of Seventh Annual Conference on the Internet Society, Kuala Lumpur, Malaysia (1997).

[38] W. Jianxin, C. Jianer, C Songqiao, An effective randomized QoS Routing algorithm on networks with inaccurate parameters, Source Journal of Computer Science and Technology 17 (2002) 33-46.

[39] X. Masip-Bruin, S. Sánchez-López, J. Solé-Pareta, J. DomingoPascual, A QoS routing mechanism for reducing the routing inaccuracy effects, Proceedings of the Second International Workshop on QoS in Multiservice IP Networks (QoS-IP 2003), Milan, Italy (2003).

[40] X. Masip-Bruin, S. Sánchez-López, J. Solé-Pareta, J. DomingoPascual, QoS routing algorithms under inaccurate routing information for bandwidth constrained applications, Proceedings of IEEE ICC 2003, Anchorage, Alaska, USA (2003). 
[41] S. Nelakuditi, Z. Zhang, R. Tsang, Adaptive proportional routing: a localized QoS routing approach, Proceedings of INFOCOM'2000, Tel-Aviv, Israel (2000).

[42] J. Shen, J. Shi, J. Crowcroft, Proactive multi-path routing, Proceedings of the International Workshop on Quality of future Internet Services (QofIS 2002), Zurich, Switzerland (2002).

[43] X. Yuan, A. Saifee, Path selection methods for localized quality of service routing, Proceedings of the 10th IEEE International Conference on Computer Communications and Networks (IC3N 2001), Phoenix, Arizona (2001).

[44] G. Apostolopoulos, R. Guérin, S. Kamat, S. Tripathi, Improving QoS routing performance under inaccurate link state information, Proceedings of the 16th International Teletraffic Congress (ITC-16), Edinburgh, UK (1999).

[45] Q. Ma, P. Steenkiste, Supporting dynamic inter-class resource sharing: a multi-class QoS routing algorithm, Proceedings of IEEE INFOCOM'99, New York, USA (1999).

[46] E. Crawley, R. Nair, B. Rajagopalan, H. Sandick, A framework for QoS-based routing in the Internet, Internet Engineering Task Force, Request for Comments 2386 (1998).

[47] M. Curado, O. Reis, J. Brito, G. Quadros, E. Monteiro, Class-pinning: a novel approach for Hop-By-Hop QoS routing, Proceedings of IASTED International Conference on Communications, Internet And Information Technology (CIIT 2002), St. Thomas, Virgin Islands, USA (2002).

[48] B. Wang, X. Su, C.L.P. Chen, A New Bandwidth Guaranteed Routing Algorithm for MPLS Traffic Engineering, Proceedings of ICC 2002, IEEE (2002) 1001-1005.

[49] J. Rekhter, 'The NSFNET Backbone SPF based Interior Gateway Protocol', RFC 1074, 1988.

[50] H. Schulzrinne, A. Rao, R. Lanphier, 'Real Time Streaming Protocol (RTSP)', RFC 2326, 1998.

[51] E. Marín-Tordera, X. Masip-Bruin, S. Sánchez-López, J. Solé-Pareta, J. Domingo-Pascual, A new prediction-based routing and wavelength assignment mechanism for optical transport networks, Proceedings of QofIS 2004, Barcelona, Catalunya, Spain (2004).

[52] P. Baran, On distributed communications networks, IEEE Transactions on Communications (1964) 1-9.

[53] T. Anjali, C. Scoglio, J. de Oliveira, L.C. Chen, I.F. Akyldiz, J.A. Smith, G. Uhl, A. Sciuto, A new path selection algorithm for MPLS networks based on available bandwidth estimation, Proceedings of QofIS 2002, Zürich, Switzerland (2002).

[54] C. Busch, M. Herlihy, R.Wattenhofer, 'Routing without Flow Control', ACM Symposium on Parallel Algorithms and Architectures 2001.

[55] A study of branch prediction strategies, Proceedings of Eigth International Symposium in Computer Architecture, Minneapolis (1981).

[56] C. Labovitz, A. Ahuja, A. Bose, F. Jahanian, Delayed Internet routing convergence, Proceedings of ACM SIGCOMM (2000).

[57] G. Cristallo, C. Jacquenet, An approach to inter-domain traffic engineering, Proceedings of XVIII World Telecommunications Congress (WTC2002), France (2002).
[58] L. Xiao, K.-S. Lui, J. Wang, K. Nahrstedt, QoS extension to BGP, IEEE ICNP (2002).

[59] K.H. Ho, N. Wang, P. Trimintzios, G. Pavlou, Traffic engineering for inter-domain quality of service, Proceedings of the London Communications Symposium (LCS) (2003).

[60] B. Quoitin, C. Pelsser, L. Swinnen, O. Bonaventure, S. Uhlig, Interdomain traffic engineering with BGP, IEEE Communications Magazine (2003).

[61] P. Pan, E. Hahne, H. Schulzrinne, BGRP: Sink-tree-based aggregation for inter-domain reservations, Journal of Communications and Networks 2 (2) (2000).

[62] L. Subramanian, I. Stoica, H. Balakrishnan, R. Katz, OverQoS: offering internet QoS using overlays, ACM SIGCOMM, Computer Communications Review (2003).

[63] Agarwal, C. Chuah, R. Katz, OPCA: robust interdomain policy routing and traffic control, IEEE Openarch (2003).

[64] Z. Li, P. Mohapatra, QRON: QoS-aware routing in overlay networks, IEEE Journal on Selected Areas in Communications (2003).

[65] Cisco Systems, Inc, 'Cisco Optimized Edge Routing Deployment Guide', white paper.

[66] M. Yannuzzi, A. Fonte, X. Masip, E. Monteiro, S. Sánchez, M. Curado, J. Domingo, A proposal for inter-domain QoS Routing based on distributed overlay entities and QBGP, Proceedings of the First International Workshop on QoS Routing (WQoSR), LNCS 3266, Barcelona, Catalunya, Spain (2004).

[67] J. Abley, B. Black, V. Gill, Goals for IPv6 site-multihoming architectures, RFC 3582, 2003.

[68] J. Hagino, H. Snyder, IPv6 multihoming support at site exit routers, RFC 3178, 2001.

[69] De Launois: http://www.ietf.org/internet-drafts/draft-de-launoismulti6-naros-01.txt.

[70] Beijnum: http://www.ietf.org/internet-drafts/draft-van-beijnummulti6-isp-int-aggr-02.txt.

[71] The Routing Information Service: http://www.ripe.net/projects/ris/ index.html.

[72] The Sixth Framework Programme: http://fp6.cordis.lu/fp6/home.cfm.

[73] Tim Griffin, 'The Stable Paths Problem as a Model of BGP Routing' http://www.cambridge.intel-research.net/ tgriffin/talks_tutorials.

[74] Tim Griffin, 'BGP wedgies' http://www.cambridge.intel-research.net/ $\sim$ tgriffin/talks_tutorials.

[75] K. Kar, M. Kodialam, T.V. Lakshman, Minimum interference routing of bandwidth guaranteed tunnels with MPLS traffic engineering applications, IEEE JSAC 18 (2000) 2566-2579 December.

[76] G. Banerjee, D. Sidhu, Comparative analysis of path computation techniques for MPLS traffic engineering, Computer Networks 40 (2002) 149-165.

[77] Iliadis, D. Bauer, A new class of online minimum-interference routing algorithms, Proceedings of NETWORKING 2002 LNCS 2345 (2002) 959-971.

[78] S. Suri, M. Waldvogel, D. Bauer, P.R. Warkhede, Profile-based routing and traffic engineering, Computer Communications 26 (2003) $351-365$. 